\title{
Draft genome sequence data and comparative analysis of Erysipelothrix Rhusiopathiae vaccine strain VR-2
}

\author{
Svetlana N. Kovalchuk ${ }^{1}$ (D) Anna V. Babii ${ }^{1}$ (D)
}

Received: 21 July 2020 / Accepted: 21 September 2020 / Published online: 29 September 2020

(c) King Abdulaziz City for Science and Technology 2020

\begin{abstract}
Erysipelothrix rhusiopathiae VR-2 is a commercially available live attenuated vaccine strain widely used in Russia, Kazakhstan, and a number of European countries for immunization of pigs against swine erysipelas. The draft genome sequence of E. rhusiopathiae strain VR-2 reported in this paper is 1,704,727 bp in length, has CG content of 36.5\%, and contains 1680 genes, including 51 tRNA, 3 rRNA, and 1408 protein-coding genes. Comparative sequence analysis between Fujisawa (serovar 1a), VR-2 and six other serovar $N$ strains of $E$. rhusiopathiae revealed wide genetic variability of the chromosomal region essential for serovar-specific antigenicity and virulence of E. rhusiopathiae strains. We have performed a BLAST search and found 12 genomic loci potentially specific for the E. rhusiopathiae VR-2 strain. These data could be helpful for developing genetic assays for differentiation of field isolates and this live attenuated vaccine strain, which is especially important for epizootical monitoring of swine erysipelas in countries, where the live vaccine strain E. rhusiopathiae VR-2 is used for pig immunization, as well as for the design of recombinant vaccines against swine erysipelas. The genome of $E$. rhusiopathiae VR-2 has been submitted in GenBank under accession number RJTK00000000.1.
\end{abstract}

Erysipelothrix rhusiopathiae is a gram-positive, non-sporeforming, rod-shaped bacterium that causes erysipeloid in humans and erysipelas in animals, including swine erysipelas (Wood, 2006). The clinical signs of swine erysipelas manifest as acute septicemia (acute phase of swine erysipelas), urticaria (subacute phase) and arthritis, lymphadenitis, and endocarditis (in the chronic stage of the disease). Due to the economic importance of swine erysipelas in the swine industry, an epizootic of E. rhusiopathiae is controlled by the administration of commercially available E. rhusiopathiae vaccines in many countries (Mansfield and Fox 2018). In Russia, Kazakhstan, and a number of European countries E. rhusiopathiae live attenuated vaccine strain

Electronic supplementary material The online version of this article (https://doi.org/10.1007/s13205-020-02451-7) contains supplementary material, which is available to authorized users.

Svetlana N. Kovalchuk

s.n.kovalchuk@gmail.com

Anna V. Babii

anna.babii@yahoo.com

1 Federal Science Center for Animal Husbandry Named After Academy Member L.K. Ernst, Dubrovitsy 60, Podolsk Municipal District 142132, Moscow Region, Russian Federation
VR-2 is widely used for immunization of pigs against swine erysipelas. The E. rhusiopathiae VR-2 strain (serovar N) was isolated in 1931 in the Pasteur Institute in Bucharest from a pig that died from swine erysipelas. The attenuated E. rhusiopathiae VR-2 strain was shown to remain highly immunogenic for pigs (Astashova, 1975; Bodurova, 1973; Majdan et al. 1972). However, the use of attenuated vaccine strains is always associated with risks of reversion to virulence that causes outbreaks of erysipelas. Therefore, the differentiation of $E$. rhusiopathiae vaccine strain VR-2 and field isolates is especially important for epizootical monitoring of swine erysipelas in countries where this commercially available live attenuated vaccine is used for immunization of pigs against swine erysipelas. In this regard, whole genome sequencing and comparative genomic analysis are promising approaches for searching the genetic markers of E. rhusiopathiae vaccine strain VR-2 to develop genetic assays for differentiation of field isolates and this live attenuated vaccine strain, as well as for the design of safe recombinant vaccines against swine erysipelas.

In this paper, we report the data on the draft genome sequence of $E$. rhusiopathiae vaccine strain VR-2 and comparative analysis of the chromosomal region, which was earlier shown to be essential for serovar-specific antigenicity and virulence of $E$. rhusiopathiae strains. The DNA sample 
of E. rhusiopathiae vaccine strain VR-2 was kindly provided by Dr. Anatoly Ya. Samuylenko from FSBSI «All-Russian Scientific Research and Technological Institute of Biological Industry» (FSBSI VNITIBP RAS, Shchelkovsky district, Moscow Region, Russia). Genomic DNA was extracted and purified with a standard phenol-chloroform method. A whole-genome shotgun fragment library was constructed from $500 \mathrm{ng}$ of genomic DNA (Rapid Library Preparation Kit; Roche) according to the manufacturer's instructions. The shotgun library was amplified by emulsion PCR (GS Junior Titanium emulsion PCR kit; Roche). The nucleotide sequence of the amplified DNA library was determined by 454 pyrosequencing (GS Junior; Roche). The sequence reads were assembled into 39 contigs of 1,704,727 bp in total length using the GS de novo Assembler v. 2.7 with default parameters. The draft genome sequence of the E. rhusiopathiae strain VR-2 was deposited in the GenBank database under accession RJTK00000000.1. The general features of the E. rhusiopathiae strain VR-2 genome sequence are summarized in Table 1. The E. rhusiopathiae strain VR-2 genome size and CG content correlate well with those of other E. rhusiopathiae strains deposited in GenBank. The genomes of $E$. rhusiopathiae strains are characterized by a relatively small size (from 1.75 to $2.9 \mathrm{Mb}$ ), which reflects the intracellular life in nutrient-rich conditions (Davis et al. 2013). Low CG content of the E. rhusiopathiae strain VR-2 genome $(36.5 \%)$ confirms the findings on negative correlation between genome size and genomic \% GC within strains of bacterial species (Bohlin et al. 2014).

The E. rhusiopathiae VR-2 genome annotation was performed using the NCBI Prokaryotic Genomes Automatic Annotation Pipeline (Tatusova et al. 2016; Haft et al. 2017). Annotation of the assembled genome of E. rhusiopathiae VR-2 revealed 1,680 genes, 1,415 proteins, 3 rRNA, 50 tRNA. According to NCBI genome neighbor report, the genome of E. rhusiopathiae VR-2 had the maximal genomic similarity (symmetric identity) with the E. rhusiopathiae WH1301, Fujisawa, and ATCC 19414 strains-94.9695, 94.7062 and $94.5322 \%$, respectively.

Virulence of E. rhusiopathiae strains varies considerably among different serovars, which are closely related to clinical forms. Among 17 known serovars assigned to the species E. rhusiopathiae (1a, 1b, 2, 4, 5, 6, 8, 9, 11, 12,

Table 1 General features of the E. rhusiopathiae strain VR-2 genome sequence

\begin{tabular}{ll}
\hline Feature & Value \\
\hline Size & $1,704,727$ \\
Coverage & $20 \times$ \\
GC content & $36.5 \%$ \\
N50 & 83,301 \\
L50 & 7 \\
Number of contigs & 39 \\
\hline
\end{tabular}

$15,16,17,19,21,23$, and $N$ ) serovars $1 \mathrm{a}, 1 \mathrm{~b}$, and 2 are frequently isolated from diseased pigs (Wood 2006). To date, mechanisms of E. rhusiopathiae virulence have not been clarified in detail. Recently, the chromosomal region essential for serovar-specific antigenicity and virulence of serovar 1 and 2 strains of E. rhusiopathiae was identified on the basis of a mutagenesis study of $15-\mathrm{kb}$ genomic region of Fujisawa strain (serovar 1a), the highly virulent strain of $E$. rhusiopathiae (Ogawa et al. 2018). This chromosomal region was revealed to encompass genes of biosynthetic pathways for polysaccharides and to be required for expression and maintenance of the molecular integrity of the capsule, which is the most important virulence factor of E. rhusiopathiae. Comparative analysis of this region from different serovars ( $1 \mathrm{a}, 1 \mathrm{~b}, 2$ and $N$ ) elucidated sequence variations, which was assumed to determine the antigenic differences among $E$. rhusiopathiae serovars (Ogawa et al. 2018; Shimoji et al. 2019).

We have identified the corresponding region in the $E$. rhusiopathiae VR-2 strain genome sequence and compared it with those of serovar 1a Fujisawa strain and serovar $\mathrm{N}$ Mie 02-47, Nagano 11-1, Ishikawa 02-26, MEW22, and Yamanashi 04-44 strains (Fig. 1). In general, significant differences in gene content of this genome region were found both between serovar 1a Fujisawa and serovars $N$ strains of E. rhusiopathiae and within the group of serovar $N$ strains (Fig. 1). E. rhusiopathiae VR-2 showed a greater similarity of this genome region with Fujisawa strain (serovar 1a) and Mie 02-47 (serovar $N$ ) than with other serovar $N$ strains. In the E. rhusiopathiae VR-2 strain this region has 17,550 bp in length (contig 4: 18,595-36,145 bp), which is about $3 \mathrm{~kb}$ longer than the corresponding region of Fujisawa and Mie 02-47 strains due to insertion of three genes, encoding NAD(P)-dependent oxidoreductase, diaminopimelate decarboxylase and sugar transferase (Fig. 1a-c letters, respectively). Besides, VR-2 strain differs from Fujisawa and Mie 02-47 strains by deletion of the gene encoding sugar transferase (ERH_1446 in Fujisawa strain) (Fig. 1, number 8 ). The same differences were found in serovar $N$ Nagano 11-1 and Ishikawa 02-26 strains (Fig. 1). Genes, corresponding to ERH_1438, ERH_1445, ERH_1447, ERH_1448, ERH_1449, ERH_1450, and ERH_1451 in Fujisawa strain, are strictly conserved in all analyzed strains (Fig. 1). The involvement of two of these genesERH_1449 and ERH_1450_in E. rhusiopathiae virulence was confirmed earlier by mutagenesis studies (Ogawa et al. 2018). Besides, the aminotransferase gene (ERH_1447 in Fujisawa) was shown to play an essential role in the antigenicity of $E$. rhusiopathiae strains. Mutagenesis studies revealed that substitution of Arg 196 in the aminotransferase of Mie 02-47 strain (serovar $N$ ) by Gly, which is characteristic in this position in the aminotransferase of Fujisawa strain (serovar 1a), recovered the antigenic reactivity of the 
Fig. 1 Gene organization of the chromosomal region essential for antigenicity and virulence of E. rhusiopathiae strains. Genes identified in all strains analyzed in this study are indicated by black arrows. Genes identified only in serovar $N$ strains are indicated by grey arrows. Identical genes are indicated by the same numbers or letters. The number in parentheses after the strain name indicates the serovar

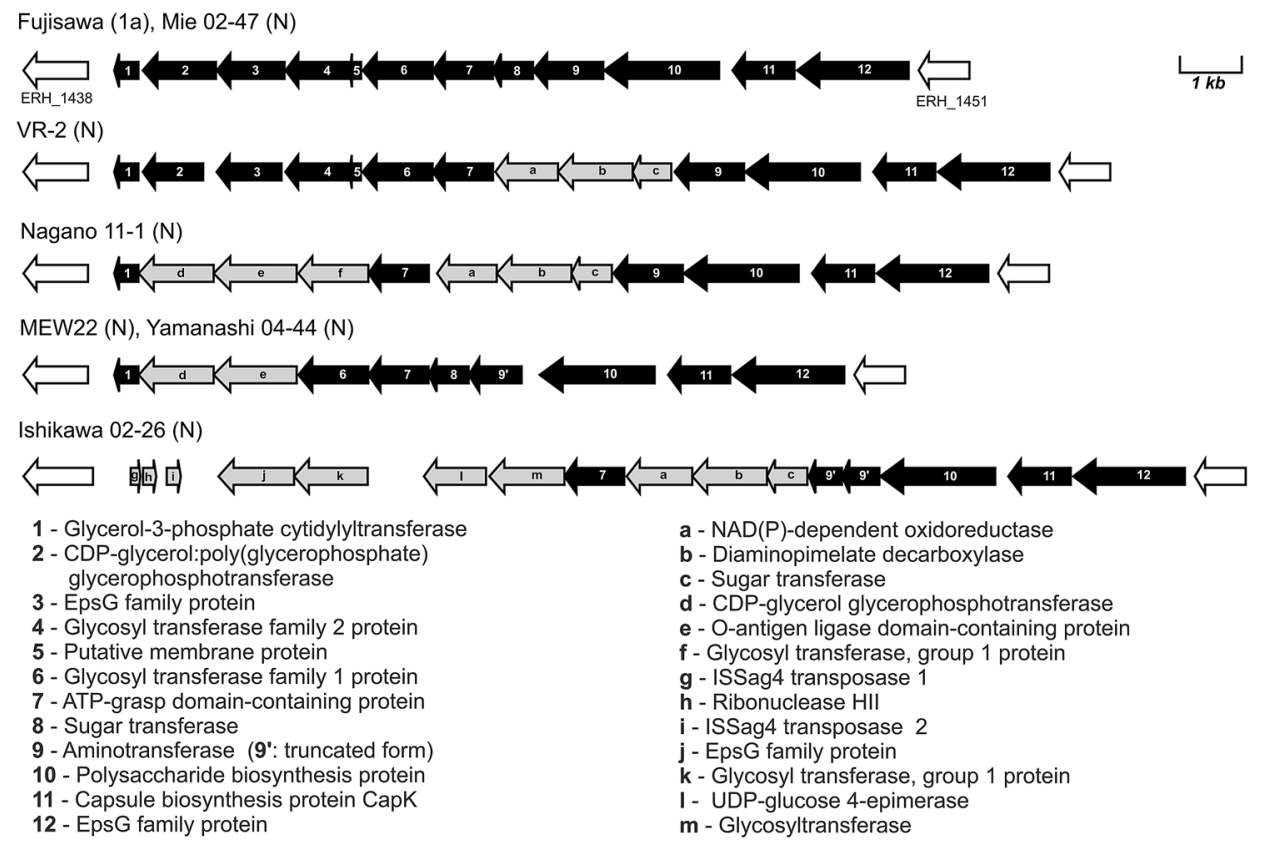

mutant Mie 02-47/C strain with serovar 1a-specific rabbit serum (Ogawa et al. 2018). Moreover, the introduction of the truncated aminotransferase gene from Ishikawa 02-26 ( $\operatorname{serovar} N$ ) into Tochigi-20 strain (serovar 2) resulted in the loss of antigenic reactivity of Tochigi-20 with serovar 2-specific rabbit serum (Ogawa et al. 2018). These results show that changes in the aminotransferase structure effect on the antigenicity of E. rhusiopathiae strains. We have compared amino acid sequences of the aminotransferase from Fujisawa (ERH_1447) and homologs in the $N$ serovar strains of $E$. rhusiopathiae and revealed that this substitution is characteristic only for Mie 02-47 strain. This fact allows us to assume that this mutation in the aminotransferase is not responsible for the antigenicity of E. rhusiopathiae stains on its own. Besides, we have found 9 novel amino acid substitutions at C-terminus of the aminotransferase in VR-2, Nagano 11-1 and Ishikawa 02-26 (data not shown). The effects of the identified amino acid sequence variants of this enzyme on the antigenicity of E. rhusiopathiae strains will be of interest for further research.

The main feature of vaccines is their immunogenicity. It has been reported that surface protective antigen proteins (Spa), localized at the bacteria cell surfaces, are the major components protecting pigs against swine erysipelas (Makino et al. 1998; Imada et al. 1999; Shimoji 2000; Shimoji et al. 1999). The Spa proteins of E. rhusiopathiae are classified into 3 molecular types, named SpaA, SpaB, and SpaC, based on their amino acid sequence similarity (To and Nagai 2007). The SpaA, SpaB, and SpaC proteins differ in the antigenic property (Shimoji et al. 2019). The data on the influence of deleting spaA gene from the genome of $E$. rhusiopathiae strain $\mathrm{C} 43065$ on the virulence of this strain
(Borrathybay et al. 2015) as well as the results of the comparative proteomic and transcriptomic analysis conducted by Li et al. (2016) for highly virulent HX130709 strain and its isogenic avirulent derivative HX130709a confirmed the association of $\mathrm{SpaA}$ with the virulence of E. rhusiopathiae and an important role of this protein in the pathogenesis of swine erysipelas. Vaccines against swine erysipelas are mainly derived from SpaA-possessing E. rhusiopathiae strains of serovars 1 and 2 (Shimoji et al. 2019; To and Nagai 2007). E. rhusiopathiae live attenuated vaccine strain VR-2 belongs to serovar N (Astashova 1975; Bodurova 1973; Majdan et al. 1972). E. rhusiopathiae VR-2 genome sequence analysis revealed the presence of spaA gene (locus EF876_RS08285), which correlated with data on the association of SpaA with E. rhusiopathiae strains of serovar N, as well as $1 \mathrm{a}, 1 \mathrm{~b}, 2,5,8,9,12,15-17$ and 23 (To and Nagai 2007). However recent studies revealed that it is not a strict rule and disassociation of the Spa types and serovars of E. rhusiopathiae strains can occur in swine isolates (Shimoji et al. 2019). SpaA protein structure is highly conserved among SpaA-possessing strains. The identity of SpaA from E. rhusiopathiae VR-2 with corresponding sequences from GenBank was from 97 to $100 \%$.

We have performed a BLAST search to identify genomic regions potentially specific for the E. rhusiopathiae VR-2 vaccine strain. As a result, eleven genes and one intergenic region were found which had no significant similarity with any other sequences from BLAST databases (Table 2). Seven of these genes are located in the contig 2 (from 17,573 to $26,383 \mathrm{bp}$ ) and encode the DNA (cytosine-5-)methyltransferase, two PD-(D/E)XK motif proteins, three hypothetical proteins with unknown functions, and an

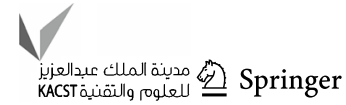


Table 2 Potential VR-2—specific genome loci

\begin{tabular}{lllll}
\hline Location & Gene locus tag & Region (bp) & Length (bp) & Description \\
\hline contig00002 & EF876_01155 & $17,573-18,799$ & 1227 & DNA (cytosine-5-)-methyltransferase/protein_id=RNM31889.1 \\
contig00002 & EF876_01160 & $18,818-19,315$ & 498 & PD-(D/E)XK motif protein/protein_id=RNM31890.1 \\
contig00002 & EF876_01165 & $19,315-19,719$ & 405 & PD-(D/E)XK motif protein/protein_id=RNM31891.1 \\
contig00002 & EF876_01170 & $19,733-20,254$ & 522 & Hypothetical protein/protein_id=RNM31892.1 \\
contig00002 & EF876_01175 & $20,429-21,376$ & 948 & Hypothetical protein/protein_id=RNM31893.1 \\
contig00002 & EF876_01180 & $21,376-23,415$ & 2040 & Hypothetical protein/protein_id=RNM31894.1 \\
contig00002 & EF876_01185 & $23,507-26,383$ & 2877 & Endonuclease/protein_id=RNM31895.1 \\
contig00003 & - & $89,461-90,150$ & 690 & Intergenic region between EF876_02470 and EF876_02475 \\
contig00011 & EF876_05525 & $20,591-21,097$ & 506 & DUF4352 domain-containing protein/protein_id=RNM29213.1 \\
contig00011 & EF876_05530 & $21,321-22,235$ & 915 & Exonuclease/protein_id=RNM29214.1 \\
contig00011 & EF876_05550 & $23,531-23,753$ & 201 & KTSC domain-containing protein/protein_id=RNM29218.1 \\
contig00011 & EF876_05585 & $29,011-29,940$ & 930 & DUF4145 domain-containing protein /protein_id=RNM29225.1 \\
\hline
\end{tabular}

endonuclease. The PD-(D/E)XK proteins constitute a functionally diverse superfamily that is involved in nucleic acids modification processes. For instance, PD-(D/E) XK domain occurs in all classes of restriction enzymes, which together with methyltransferases set up the restriction-modification systems of bacterial and archaeal cells. Multiple horizontal gene transfer events for PD-(D/E)XK proteins were revealed earlier between human pathogenic bacteria or from Prokaryota to Eukaryota (Steczkiewicz et al. 2012). Taking into consideration the fact of absence of these genes in other 15 known genomes of E. rhusiopathiae strains and their successive localization in the $E$. rhusiopathiae VR-2 genome, it could be hypothesized that this genomic region has arisen in E. rhusiopathiae VR-2 strain by horizontal gene transfer from another bacterial species. Other genomic regions potentially specific for $E$. rhusiopathiae VR-2 strain are located in contig 11 and encodes DUF4352 domain-containing protein, an exonuclease, KTSC domain-containing protein and DUF4145 domain-containing protein (Table 2). According to Pfam annotation, DUF4352 domain is immunoglobulin-like domain which was found in proteins that fall into the antigen MPT63/MPB63 (immunoprotective extracellular protein) superfamily. DUF4145 domain was found in a variety of restriction endonuclease enzymes, but the exact functions of this domain as well as KTSC domain are uncertain. These genomic regions identified as potentially specific for E. rhusiopathiae VR-2 strain could be used for developing DNA-based assays aimed to differentiate this vaccine strain from field isolates.

Accession numbers This Whole Genome project has been deposited into GenBank under the accession no. RJTK00000000. The version described in this paper is version RJTK00000000.1.
Acknowledgments The study was funded by the Ministry of science and higher education of the Russian Federation (№ 075-01250-20-01). We would like to thank Dr. Anatoly Ya. Samuylenko from FSBSI «AllRussian Scientific Research and Technological Institute of Biological Industry» (FSBSI VNITIBP RAS, Shchelkovsky district, Moscow Region, Russia) for kindly providing us with the genomic DNA sample of E. rhusiopathiae VR-2.

Author contributions SNK and AVB contributed equally to this work.

\section{Compliance with ethical standards}

Conflict of interest The authors declare that they have no conflict of interest in the publication.

\section{References}

Astashova EA (1975) Morphocytochemical study of Erysipelothrix rhusiopathiae of the vaccine strain VR-2. Veterinariia 8:29-30

Bodurova T (1973) Properties of the lyophilized anti-swine-erysipelas vaccine (VR2). Vet Med Nauk 10:65-69

Bohlin J, Sekse C, Skjerve E, Brynildsrud O (2014) Positive correlations between genomic \% AT and genome size within strains of bacterial species. Environ Microbiol Rep 6:278-286. https://doi. org/10.1111/1758-2229.12145

Borrathybay E, Gong FJ, Zhang L, Nazierbieke W (2015) Role of surface protective antigen A in the pathogenesis of Erysipelothrix rhusiopathiae strain C43065. J Microbiol Biotechnol 25:206-216. https://doi.org/10.4014/jmb.1407.07058

Davis JJ, Xia F, Overbeek RA, Olsen GJ (2013) Genomes of the class Erysipelotrichia clarify the firmicute origin of the class Mollicutes. Int J Syst Evol Microbiol 63:2727-2741. https://doi. org/10.1099/ijs.0.048983-0

Haft DH, DiCuccio M, Badretdin A et al (2017) RefSeq: an update on prokaryotic genome annotation and curation. Nucleic Acids Res 46:D851-D860. https://doi.org/10.1093/nar/gkx1068

Imada Y, Goji N, Ishikawa H et al (1999) Truncated surface protective antigen (SpaA) of Erysipelothrix rhusiopathiae serotype $1 \mathrm{a}$ elicits protection against challenge with serotypes $1 \mathrm{a}$ and $2 \mathrm{~b}$ 
in pigs. Infect Immun 67:4376-4382. https://doi.org/10.1128/ iai.67.9.4376-4382.1999

Li Y, Zou Y, Xia Y et al (2016) Proteomic and transcriptomic analyses of swine pathogen Erysipelothrix rhusiopathiae reveal virulence repertoire. PLoS ONE 11:e0159462. https://doi.org/10.1371/journ al.pone. 0159462

Majdan S, Krzyszowski M, Kocik T (1972) Anti-erysipelas vaccine from the VR2 strain. Veterinariia 9:116-118

Makino SI, Yamamoto K, Murakami S et al (1998) Properties of repeat domain found in a novel protective antigen, SpaB, of Erysipelothrix rhusiopathiae. Microb Pathog 25:101-109. https://doi. org/10.1006/mpat.1998.0216

Mansfield KG, Fox JG (2018) Bacterial diseases. In: Marini R, Wachtman L, Tardif S, et al. (eds) The Common Marmoset in Captivity and Biomedical Research. Elsevier, pp 265-287

Ogawa Y, Shiraiwa K, Nishikawa S et al (2018) Identification of the chromosomal region essential for serovarspecific antigen and virulence of serovar 1 and 2 strains of Erysipelothrix rhusiopathiae. Infect Immun 86:e00324-e418. https://doi.org/10.1128/IAI.00324 $-18$

Shimoji Y (2000) Pathogenicity of Erysipelothrix rhusiopathiae: virulence factors and protective immunity. Microbes Infect 2:965-972

Shimoji Y, Bito M, Shiraiwa K et al (2019) Disassociation of Spa type and serovar of an Erysipelothrix rhusiopathiae serovar 6 strain isolated from a diseased pig. J Vet Diagn Investig 31:488-491. https://doi.org/10.1177/1040638719835883

Shimoji Y, Mori Y, Fischetti VA (1999) Immunological characterization of a protective antigen of Erysipelothrix rhusiopathiae: Identification of the region responsible for protective immunity. Infect Immun 67:1646-1651. https://doi.org/10.1128/ iai.67.4.1646-1651.1999

Steczkiewicz K, Muszewska A, Knizewski L, Rychlewski L, Ginalski K (2012) Sequence, structure and functional diversity of PD-(D/E)XK phosphodiesterase superfamily. Nucleic Acids Res 40(15):7016-7045. https://doi.org/10.1093/nar/gks382

Tatusova T, DiCuccio M, Badretdin A et al (2016) NCBI prokaryotic genome annotation pipeline. Nucleic Acids Res 44:6614-6624. https://doi.org/10.1093/nar/gkw569

To H, Nagai S (2007) Genetic and antigenic diversity of the surface protective antigen proteins of Erysipelothrix rhusiopathiae. Clin Vaccine Immunol 14:813-820. https://doi.org/10.1128/CVI.00099 $-07$

Wood LR (2006) Erysipelas. In: Straw BE, Zimmermann JJ, D’Allaire S, Taylor DJ (eds) Diseases of swine, 9th edn. Blackwell Publishing Professional, Ames, pp 629-638 